\title{
Dissecting the Links Between Cerebellum and Dystonia
}

\author{
Ailish Malone • Mario Manto • Chris Hass
}

Published online: 20 September 2014

(C) Springer Science+Business Media New York 2014

\begin{abstract}
Dystonia is a common movement disorder characterized by sustained muscle contractions. These contractions generate twisting and repetitive movements or typical abnormal postures, often exacerbated by voluntary movement. Dystonia can affect almost all the voluntary muscles. For several decades, the discussion on the pathogenesis has been focused on basal ganglia circuits, especially striatal networks. So far, although dystonia has been observed in some forms of ataxia such as dominant ataxias, the link between the cerebellum and dystonia has remained unclear. Recent human studies and experimental data mainly in rodents show that the cerebellum circuitry could also be a key player in the pathogenesis of some forms of dystonia. In particular, studies based on behavioral adaptation paradigm shed light on the links between dystonia and cerebellum. The spectrum of movement disorders in which the cerebellum is implicated is continuously expanding, and manipulation of cerebellar circuits might even emerge as a candidate therapy in the coming years.
\end{abstract}

Dystonia has traditionally been causally associated with lesions in the basal ganglia. Much of this understanding is based on the findings of overt basal ganglia lesions in neuroimaging and autopsy studies [1]. This localization of dystonia to the basal ganglia does not accommodate a growing body of

\footnotetext{
A. Malone

Gait Laboratory, Central Remedial Clinic, Clontarf Dublin 3, Ireland

M. Manto $(\bowtie)$

Unité d'Etude du Mouvement ULB-Erasme, 808 Route de Lennik, 1070 Bruxelles, Belgium

e-mail:mmanto@ulb.ac.be

C. Hass

Center for Movement Disorders and Neurorestoration, University of Florida, Gainesville, FL, USA
}

clinical and experimental evidence of the involvement of other structures, and the concept of dystonia as a network disorder involving multiple brain regions has been proposed $[2,3]$. The role of the cerebellum in the pathophysiology of dystonia was first noted by Fletcher et al. [4] and there is strong evidence of abnormal cerebellar function from pathological, neuroimaging, and neurophysiological studies $[2,3,5]$, particularly in forms of primary dystonia [6-8].

There is little doubt then that the cerebellum is involved in dystonia, but there has been much debate as to whether the cerebellum plays a role in the primary pathological process, or whether the abnormalities are secondary features to compensate for deficient basal ganglia functioning. Neychev et al. [3] discuss the concept that irritative lesions to the cerebellum, which do not involve loss of cerebellar tissue but rather distort cerebellar output, can lead to dystonia. These lesions are likely to be focal hemorrhages or space-occupying lesions that compress and distort cerebellar functions, leading to an increase rather than a decrease in blood flow to the cerebellum $[3,9]$. The hypothesis is consistent with the relationship between dystonia and tremor because tremor is also viewed as a distortion of cerebellar functioning [2]. Neuroimaging studies have consistently shown above-normal cerebellar activation in dystonia [3]. While these findings are compelling, research defining the extent and nature of cerebellar involvement in patients with dystonia is incomplete.

Rodent models such as the tottering mouse, the dystonic rat, and the model of rapid onset dystonia parkinsonism are also contributing to a better understanding of the links between cerebellum and dystonia [10]. Electrophysiological studies in dystonic mice reveal abnormal high-frequency bursting activity in cerebellar nuclei, the sole output of cerebellar circuitry [11]. The activity appears as aberrant. The firing patterns of Purkinje neurons are also impaired. The sodium pump seems to play a critical role as shown by in vitro experiments. Interestingly, partial cerebellectomy 
may improve symptoms, providing another strong argument for a cerebellar role in dystonia [10]. The observed combination of reduced activity of Purkinje neurons and increased intrinsic excitability of cerebellar nuclei opens novel avenues for pharmacological therapies of dystonia [10].

A new study [12] extends the investigation of the role of the cerebellum in primary dystonia beyond the more pure forms of cerebellum-dependent associative motor learning, such as eye-blink classical conditioning, to motor adaptation paradigms. Within traditional motor adaptation paradigms, adaptive learning is assessed by the degree of the after-effect observed during a de-adaptation task and the amount of savings upon a second exposure to the adaptation task (i.e., a readaptation task). In context of the present study, locomotor adaptation is a process during which changes in locomotor output are stabilized over time by the central nervous system's incorporation of feed-forward predictive motor actions and sensorimotor feedback [13-19]. While locomotor adaptation has previously been induced within the laboratory setting using a variety of different methods, Hoffland et al. utilize a locomotor adaptation paradigm using a split-belt treadmill. With split-belt treadmills, the two belts can be decoupled such that one leg walks faster than the other. During adaptation to the split belt treadmill perturbation, intralimb spatiotemporal variables (e.g., stride length and stance time) are reactively altered by feedback mechanisms likely derived from the spinal cord $[20,21]$ while interlimb parameters (e.g., step length and double limb support time) require cerebellar-modulated predictive, feed-forward control to more gradually adapt [19]. As the cerebellum exerts a tonic inhibitory influence over the cortex, previous research has suggested that depression of cerebellar inhibition over the motor cortex is required to facilitate the storage of aftereffects following split belt treadmill walking [18].

In the present investigation, Hoffland explores the hypothesis that different adaptive learning may exist across three forms of dystonia (blepharospasm, writer's cramp, and cervical dystonia), suggesting that different networks may exist for different types of dystonia [2,3]. During baseline walking conditions, individuals with blepharospasm and cervical dystonia showed impairment of gait demonstrated by a slower preferred walking speed and reduced balance confidence compared to healthy controls and people with writer's cramp. This can be directly attributed to the effect of involuntary neck and head movements on postural stability and vestibular input in cervical dystonia, and eye closure on visual input in blepharospasm, both of which are essential for dynamic balance during gait [22]. However, the findings of the motor learning paradigm were independent of these baseline gait deficits. The adaptation rate to the split-belt paradigm was slower in blepharospasm and writer's cramp compared to healthy controls, indicating impaired encoding of a new locomotor pattern by the cerebellum. This slowed adaptation leads to a reduction in the amount of practice of the new walking pattern leading to a reduced after-effect (storage of the new pattern) in those with blepharospasm and writer's cramp. Those with cervical dystonias performed similarly to healthy controls.

These findings expound upon the network theory of dystonia and provide further insight into the role of the cerebellum in different types of primary dystonia. Despite the fact that cervical dystonia had a direct effect on postural stability and balance in gait at baseline, reactive adaptation and anticipatory encoding of a new motor pattern were, in fact, intact for this gait-related task. People with writer's cramp had no baseline gait deficit when compared to controls, as expected given the remoteness of the affected site to the performance of gait, but showed impaired motor learning in the split-belt paradigm, as did people with blepharospasm. The impairment manifested in different ways for these two forms of dystonia. In writer's cramp, a significantly slower rate of adaptation was evident in temporal parameters (double support time and phasing or step time), whereas slower adaptation in blepharospasm was consistent with abnormalities in both spatial and temporal domains. The authors suggest an anatomical explanation in that the pontocerebellum could play a stronger role in the spatial control of locomotion whereas the spinocerebellum might be more involved in temporal aspects of locomotor control. This provides theoretical localisation of the functional disruption in cerebellar function in dystonia.

Cervical dystonia is often thought to have a clear causal link to the cerebellum. In a review of 25 secondary cervical dystonia cases, the most common causes were cerebellar lesions, whereas basal ganglia lesions accounted only for about a quarter of the cases [23]. Posterior fossa tumors are a common causative factor for cervical dystonia [5]. Abnormal motor learning in cervical dystonia was previously shown in a computer-based upper limb target task [24] and in eyeblink conditioning [25] suggesting that cerebellar function is certainly impaired. However, the results of the Hoffland study and recent work by Sadnicka et al. [26] should temper these assumptions. Sadnicka et al. investigated the adaptation in patients with cervical dystonia to both visuomotor (distorting visual feedback) and force field (applying a velocity-dependent force) perturbations of arm movements using a robotic manipulandum. The authors observed similar rates of learning and retention in both patients and controls suggesting motor adaptation in cervical dystonia is similar to healthy controls in two tasks which test visual and proprioceptive sensorimotor integration. These findings support to a large extent the observed response to split belt treadmill walking in this group of patients.

In the case of writer's cramp, the slower adaptation rates in Hoffland's study are in line with the findings of Hubsch et al. [8] who compared the performance of 21 people with writer's cramp and 25 healthy controls in a reaching task that included a visuomotor conflict. They found that people with writer's 
cramp exhibited a complete loss of both inhibitory and excitatory cerebellar priming of cortical sensorimotor plasticity. In a visuomotor task, people with writer's cramp were also less efficient than healthy subjects at washing out a previous adaptation strategy [8]. People with focal hand dystonia also showed abnormal associative learning in an eyeblink classical conditioning paradigm [24]. In the present study, however, those with writer's cramp exhibited relatively less storage of the learned pattern (carry over into tied walking, i.e., less asymmetry at the beginning of postadaptation) and were more symmetric at the end of postadaptation compared to the controls and cervical dystonia groups. This shows that the cerebellar motor learning impairment is not just localized to tasks requiring motor control of the involved dystonic segment, but may be influenced by the mode of motor learning paradigms.

The contribution of the disruption of normal cerebellar function to the pathophysiology and behavioral deficits of dystonia is a prominent focus of recent research. Hoffland et al. extend this line of research in two important ways. First, the authors rely on a motor adaptation paradigm that not only requires the cerebellum but additionally a more widespread cerebral sensorimotor network. Indeed, robust sensorimotor integration is needed to both learn the new walking pattern and to maintain dynamic stability during split-belt treadmill walking. Second, Hoffland et al. studied various types of focal dystonia to evaluate the convergence or divergence of pathophysiological mechanisms in these different subtypes. In this initial work, locomotor adaptive learning was preserved in cervical dystonia whereas abnormal sensorimotor adaptation was documented in patients with blepharospasm and writer's cramp. However, these interpretations should be tempered due to the methodological limitations of not considering sidedness when assigning legs to the fast belt as well as not controlling for absolute differences in belt speed across groups. None the less, these findings provide implications for current anatomical models of the pathophysiology across dystonia. Thus, the development of more specific evidence-based models of cerebellar dysfunction may be needed.

\section{References}

1. Marsden CD, Obeso JA, Zarranz JJ, Lang AE. The anatomical basis of symptomatic hemidystonia. Brain. 1985;108:463-83.

2. Prudente CN, Hess EJ, Jinnah HA. Dystonia as a network disorder: what is the role of the cerebellum? Neuroscience. 2014;260:23-35.

3. Neychev VK, Gross RE, Lehericy S, Hess EJ, Jinnah HA. The functional neuroanatomy of dystonia. Neurobiol Dis. 2011;42: 185-201.

4. Fletcher NA, Stell R, Harding AE, Marsden CD. Degenerative cerebellar ataxia and focal dystonia. Mov Disord. 1988;3:336-42.

5. Filip P, Lungu OV, Bares M. Dystonia and the cerebellum: a new field of interest in movement disorders? Clin Neurophysiol. 2013;124:1269-76.
6. Sadnicka A, Hoffland BS, Bhatia KP, Van De Warrenburg BP, Edwards MJ. The cerebellum in dystonia - help or hindrance? Clin Neurophysiol. 2012;123:65-70.

7. Sadnicka, A., Teo, J. T., Kojovic, M., Parees, I., Saifee, T. A., Kassavetis, P., et al. All in the blink of an eye: new insightinto cerebellar and brainstem function in DYT1 and DYT6 dystonia. Eur J Neurol. 2014, in press.

8. Hubsch C, Roze E, Popa T, Russo M, Balachandran A, Pradeep S, et al. Defective cerebellar control of cortical plasticity in writer's cramp. Brain. 2013;136:2050-62.

9. Zoons E, Booij J, Nederveen AJ, Dijk JM, Tijssen MA. Structural, functional and molecular imaging of the brain in primary focal dystonia - a review. Neuroimage. 2011;56:1011-20.

10. Luna-Cancalon K, Sikora KM, Pappas SS, Singh V, Wulff H, Paulson HL, et al. Alterations in cerebellar physiology are associated with a stiff-legged gait in Atcay(ji-hes) mice. Neurobiol Dis. 2014;67:140-8

11. Fremont R, Calderon DP, Maleki S, Khodakhah K. Abnormal highfrequency burst firing of cerebellar neurons in rapid-onset dystoniaparkinsonism. J Neurosci. 2014;34:11723-32.

12. Hoffland BS, Veugen LC, Janssen MMHP, Pasman JW, Weerdesteyn V, van deWarrenburg BP (2014). A gait pattern reveals different patterns of abnormalcerebellar motor learning in primary focal dystonias. Cerebellum. This issue

13. Purzner J, Paradiso GO, Cunic D, Saint-Cyr JA, Hoque T, Lozano $\mathrm{AM}$, et al. Involvement of the basal ganglia and cerebellar motor pathways in the preparation of self-initiated and externally triggered movements in humans. J Neurosci. 2007;27:6029-36.

14. Cavaco S, Anderson SW, Correia M, Magalhaes M, Pereira C, Tuna A, et al. Task specific contribution of the human striatum to perceptual-motor skill learning. J Clin Exp Neuropsychol. 2011;33: 51-62.

15. Bédard P, Sanes JN. Basal ganglia-dependent processes in recalling learned visualmotor adaptations. Exp Brain Res. 2011;209:385-93.

16. Bares M, Lungu O, Liu T, Waechter T, Gomez CM, Ashe J. Impaired predictive motor timing in patients with cerebellar disorders. Exp Brain Res. 2007; 180:355-65.

17. Bares M, Lungu OV, Husárová I, Gescheidt T. Predictive motor timing performance dissociates between early diseases of the cerebellum and Parkinson's disease. Cerebellum. 2010;9:124-35.

18. Jayaram G, Galea JM, Bastian AJ, Celnik P. Human locomotor adaptive learning is proportional to depression of cerebellar excitability. Cereb Cortex. 2011;21:1901-9.

19. Morton SM, Bastian AJ. Cerebellar contributions to locomotor adaptations during splitbelt treadmill walking. J Neurosci. 2006;26: 9107-16.

20. Rossignol S, Drew T, Brustein E, Jiang W. Locomotor performance and adaptation after partial or complete spinal cord lesions in the cat. Prog Brain Res. 1999;123:349-65.

21. Pearson KG. Proprioceptive regulation of locomotion. Curr Opin Neurobiol. 1995;5:786-91.

22. Shumway-Cook A, Woollacott M. Motor control: theory and practical applications. Baltimore: Williams \& Wilkins; 1995.

23. Ledoux MS, Brady KA. Secondary cervical dystonia associated with structural lesions of the central nervous system. Mov Disord. 2003;18:60-9.

24. Filip, P., Lungu, O. V., Shaw, D. J., Kasparek, T. \& Bares, M. The mechanisms of movement control and time estimation in cervical dystonia patients. Neural Plast 2013, 908741.

25. Teo JT, Van De Warrenburg BP, Schneider SA, Rothwell JC, Bhatia KP. Neurophysiological evidence for cerebellar dysfunction in primary focal dystonia. J Neurol Neurosurg Psychiatry. 2009;80:80-3.

26. Sadnicka A, Patani B, Saifee TA, Kassavetis P, Pareés I, Korlipara P, et al. Normal motor adaptation in cervical dystonia: a fundamental cerebellar computation is intact. Cerebellum. 2014;13:558-67. 